\title{
In vitro apoptotic potential of chrysin on TRAIL/TNF-alpha sensitize human lung cancer cells
}

\author{
Syed Hassan Mehdi*, Md. Zafaryab, Md. Asad Khan, Zubair Bin Hafeez, Zafar Iqbal \\ and M. Moshahid A. Rizvi ${ }^{\#}$
}

Genome Biology Lab, Department of Biosciences, Jamia Millia Islamia, New Delhi-110025

Email:*saeedimam@gmail.com,\# rizvi_ma@yahoo.com

Chrysin (5,7-dihydroxyflavone) is a natural flavonoid found in many plant extracts, including blue passion flower (Passiflora caerulea) [1-2]. Chrysin has multiple biological activities, such as anti-inflammatory, antioxidative and anti-cancer. It has already been reported to induce apoptosis in a panel of cancer cell lines, HeLa, U937 and HL-60.

The aim of this study was to evaluate Chrysin as apoptotic inducer against TRAIL/TNF-alpha sensitized Human lung cancer cell lines L-132 and A549.

Cytotoxic effect of Chrysin was evaluated through the MTT and NR assays. Apoptotic induction was demonstrated by Fluorescence microscopy through DAPI and PI staining and Western blot through specific monoclonal antibody.

Cytotoxicity was monitored through MTT as well as NR assays and the result demonstrated that Chrysin was significantly $(p<0.005)$ killed different types of Lung cancer cells, i:e, L-132 and A549. Pro-apoptotic activity was evaluated by western blot for Caspase 3/8/9, Bcl2 and Bax
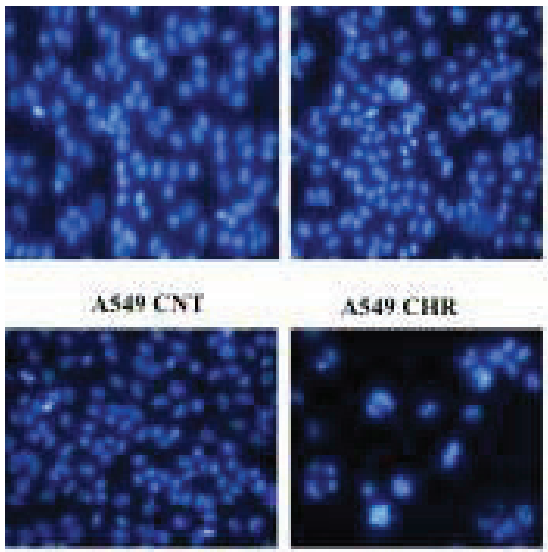

L-132 CVT proteins. Our result demonstrated that TRAIL/TNF-alpha alone could not significantly induce apoptosis, however, TRAIL/TNF-alpha along with Chrysin did induce apoptosis through activation of Caspase 3/9/8 and Bax with simultaneouly decreased expression of $\mathrm{Bcl}-2$ proteins. Further, it was confirmed through the two nuclear staining dyes DAPI and PI where cell nuclei showed altered nuclear morphology (nuclear condensation, nuclear blebbing, nuclear fragmentation) Figure 1.

The study suggested the efficacy and consequence of Chrysin in cancer therapy as it established a strong pro-apoptotic behaviour against vital human cancer cells.

Acknowledgements: Financial support from UGC D.S Kothari is acknowledged.

\section{References}

1. C.A., Williams, J.B., Harborne, M. Newman, J. Greenham, J. Eagles. Phytochemistry. 1997, 46, 1349-53.

2. X. Li, Q. Huang, C.N., Ong, X.F., Yang, H.M., Shen. Cancer Lett. 2010, 293(1), 109-16.
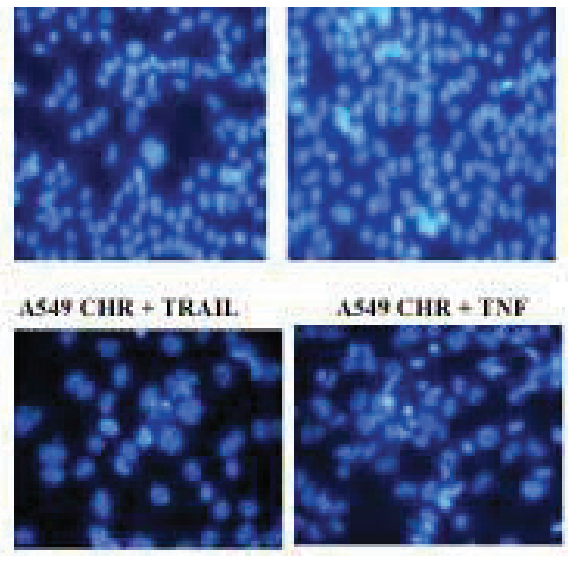

L-132 CHR + TNF

Figure 1: Nuclear morphology capture through fluorescence microscope after staining with DAPI at $20 \mathrm{X}$ 